\section{Ethnic variations in pathways to and use of specialist mental health services in the UK}

\author{
Systematic review
}

\section{KAMALDEEP BHUI, STEPHEN STANSFELD, SALLY HULL, STEFAN PRIEBE, FUNKE MOLE and GENE FEDER}

\author{
Background Inequalities of service use \\ across ethnic groups are important to \\ policy makers, service providers and \\ service users.
}

\begin{abstract}
Aims To identify ethnic variations in pathways to specialist mental health care, continuity of contact, voluntary and compulsory psychiatric in-patient admissions; to assess the methodological strength of the findings.
\end{abstract}

\begin{abstract}
Method A systematic review of all quantitative studies comparing use of mental health services by more than one ethnic group in the UK. Narrative analysis supplemented by meta-analysis, where appropriate.
\end{abstract}

Results Most studies compared Black and White patients, finding higher rates of in-patient admission among Black patients. The pooled odds ratio for compulsory admission, Black patients compared with White patients, was 4.31 (95\% Cl 3.33-5.58). Black patients had more complex pathways to specialist care, with some evidence of ethnic variations in primary care assessments.

\section{Conclusions There is strong evidence} of variation between ethnic groups for voluntary and compulsory admissions, and some evidence of variation in pathways to specialist care.

Declaration of interest None. Funding from London National Health Service Research and Development.
The Human Rights Act 1998 and the Race Relations (Amendment) Act 2000 place responsibilities on service providers to eradicate discriminatory procedures and practice. Inequitable variation in use of health care services between ethnic groups concerns policy makers, patients and clinicians (Acheson, 1998; Department of Health, 2000). A previous review reported that mental health services are unattractive to some ethnic groups, who complain of more coercive treatments and adverse experiences (Cochrane \& Sashidharan, 1996). Although this perception has become widespread, studies that compare access and use of mental health services by different ethnic groups have not been reviewed systematically. It is not known how consistently the findings suggest poorer access for minority ethnic groups, or how these findings relate to study design and methodological quality (Cochrane \& Sashidharan, 1996). We undertook a review of the published studies on ethnic variations in use of primary care or mental health services in the UK.

\section{METHOD}

Our review focused on specific questions: is there variation between ethnic groups in (a) pathways to specialist mental health care; (b) continuing contact with mental health services; (c) use of in-patient services; and (d) compulsory admissions. We included published papers that compared access to, or use of, primary or secondary mental health care in the UK by at least two ethnic groups with psychotic and non-psychotic mental disorders in primary and secondary care (see Appendix). We excluded specialist services for children, adolescents and older people, as well as forensic services and services for psychosexual disorders or substance misuse.
The following bibliographic databases were searched: ASSIA, CINAHL, the Cochrane Trials Register, EMBASE, HealthStar, Medline, PsycLIT, Science Citation Index and SIGLE. The search was restricted to studies published in English between January 1983 and October 2000 inclusive. We identified titles and abstracts of papers that potentially fulfilled our inclusion criteria. The grouped search terms included: (a) GREAT BRITAIN, HEALTH SERVICES, INDIGENOUS ETHNIC GROUP; (b) HOSPITALS, PSYCHIATRIC, MENTAL HEALTH SERVICES, PSYCHIATRY, MENTAL DISORDERS, PSYCHOTROPIC DRUGS; (c) ETHNIC or MULTI-ETHNIC, UK or BRITAIN or LONDON, MENTAL or PSYCHIATRY or PSYCHOTROPIC. In databases where MESH terms were available they were exploded and combined. Searches were adapted for the different databases and performed independently by two reviewers. These searches were supplemented by personal bibliographies of the investigators, forward citation tracking using the Science Citation Index and Social Science Citation Index databases and by references in retrieved articles. We sent a list of all included studies to three external experts, asking them to identify any other relevant studies.

Abstracts, if available, or full papers were assessed independently by two reviewers. All potentially relevant papers were then assessed against inclusion and exclusion criteria. Disagreement was resolved by a third reviewer. Data were extracted independently by two reviewers. Discrepancies were resolved by discussion or by a third reviewer. For studies reporting pathways to specialist mental health care, continuing contact with mental health services and use of in-patient services, we conducted a narrative review because differences in the measurement and reporting of outcomes made combination of point estimates inappropriate. The narrative analysis involved comparison of tabulated data and appraisal of methodological quality (see below). For the review of variation in compulsory admissions to in-patient facilities, in addition to a narrative review, we performed a meta-analysis to quantify variation between ethnic groups in compulsory admission to in-patient facilities. We have expressed the main results as combined odds ratios with the random effects method after performing tests for 
heterogeneity. We combined data on the proportions of White and Black patients who were compulsorily admitted in the primary studies. We examined publication bias and related biases in a funnel plot and carried out a test of funnel plot asymmetry. All analyses were performed with StatsDirect (http://www.statsdirect.com/).

Two reviewers independently assessed the methodological quality of each published study, with criteria adapted from Raine's review of gender differences in health service use (Table 1; Raine, 2000). We assessed the quality of published studies in four domains: sample source and size; method of ethnic group classification; adjustment for disease and confounding variables, including socio-economic status; and whether ethnic groups were combined appropriately in the analysis. Each of these was equally important in the allocation of quality score. Papers varied in their classification of ethnic groups and these were often aggregated into broad categories. In our analysis we have used the most commonly reported aggregated categories, comparing 'Black' and 'South Asian' groups with 'White' participants. We summarised the results of the papers and examined whether quality scores, geographical location of the research, the composition of the ethnic groups and study design could explain any heterogeneity of findings.

\section{RESULTS}

\section{Primary studies identified and quality scores}

A total of 545 potentially relevant titles and abstracts were identified. Sixty-seven papers were scrutinised. One duplicate publication was excluded, leaving 38 papers that met our criteria (see Appendix). One additional paper that was identified by an external reviewer did not meet our inclusion criteria. Details of each paper, including classification of ethnic groups, demographic characteristics, study design and quality ratings are summarised in Table 2 (the full review tables can be seen on the Institute of Community Health Sciences Queen Mary website at http:// www.ichs.qmul.ac.uk/transcultural/TMSPub1.htm). Quality scores ranged from 0 to 11 and were summarised into three categories: low quality (0-3); medium quality (4-7); and high quality (8-11). There were five high-quality and 23 medium-quality studies. The total quality score was most highly correlated with adjustment for confounders $(r=0.81)$ and methods for ethnic group classification $(r=0.6)$. The more recently published papers achieved higher quality scores $(r=0.57, P=0.0002)$.

\section{Methodological findings}

Most papers reported observational studies in health service settings using a crosssectional design, without making a distinction between incident and prevalent cases (see Table 2). A total of 34 studies included information from medical records, 15 from direct interview and 10 used questionnaires. Clearly the majority of studies are therefore subject to recall bias and information bias, and where samples were recruited from in-patient and out-patient services, selection bias is likely to be important. Only three studies were of a prospective cohort design in which biased assessment of risk factors is minimised. As far as could be judged, potential biases due to study design did not explain heterogeneity. Thirty-five papers included Black participants; eight did not distinguish

Table I Scoring system for methodological quality of paper

\begin{tabular}{|c|c|c|c|c|c|c|c|}
\hline \multirow[t]{2}{*}{ Sample source and size } & \multirow[t]{2}{*}{ Score } & \multirow{2}{*}{$\begin{array}{l}\text { Adjustment for } \\
\text { confounding variables }\end{array}$} & \multirow[t]{2}{*}{ Score } & \multicolumn{4}{|c|}{ Ethnicity categorisation } \\
\hline & & & & Quality & Score & Use in the analysis & Score \\
\hline $\begin{array}{l}\text { Routine data (e.g. health } \\
\text { authority, GP list data) }\end{array}$ & 0 & None & 0 & $\begin{array}{l}\text { Third-party reports (e.g. } \\
\text { ward staff categorisation, } \\
\text { name-based methods, skin } \\
\text { colour methods) }\end{array}$ & 0 & $\begin{array}{l}\text { Inappropriate ethnic groups } \\
\text { combined for major study } \\
\text { outcomes (e.g. Blacks v. all } \\
\text { others) or poor method } \\
\text { of collecting ethnicity data }\end{array}$ & 0 \\
\hline $\begin{array}{l}\text { Project-specific data: }<30 \\
\text { cases in ethnic groups for } \\
\text { major outcomes }\end{array}$ & $\mathrm{I}$ & Age and/or gender & I & $\begin{array}{l}\text { Self-reported ethnicity or } \\
\text { use of census categories }\end{array}$ & $\mathrm{I}$ & $\begin{array}{l}\text { Lumping of groups: } \\
\text { reasonable combinations } \\
\text { of groups collected by } \\
\text { census/self-report method }\end{array}$ & $\mathrm{I}$ \\
\hline $\begin{array}{l}\text { Project-specific data: }>30 \\
\text { cases in ethnic groups for } \\
\text { major outcomes }\end{array}$ & 2 & $\begin{array}{l}\text { Diagnosis or disease severity } \\
\text { (give I point if this sample } \\
\text { is selected by diagnosis) }\end{array}$ & $\mathrm{I}$ & & & $\begin{array}{l}\text { All analysis done on ethnic } \\
\text { groups without } \\
\text { amalgamation, and self- } \\
\text { report/census categories } \\
\text { for categorisation }\end{array}$ & 2 \\
\hline $\begin{array}{l}\text { Project-specific data: total } \\
\text { sample size }>500\end{array}$ & 3 & $\begin{array}{l}\text { Comorbidity and risk factors } \\
\text { for outcome of interest }\end{array}$ & $\mathrm{I}-3$ & & & & \\
\hline Maximum possible ${ }^{2}$ & 3 & & 5 & & I & & 2 \\
\hline
\end{tabular}

I. Risk factors included socio-economic factors (deprivation score, employment, household size, marital status); comorbidity included drug and alcohol use, coexisting I. Risk factors included socio-economic

2. Maximum possible score for all items=II; low quality 0-3; moderate quality 4-7; high quality 8-II. 


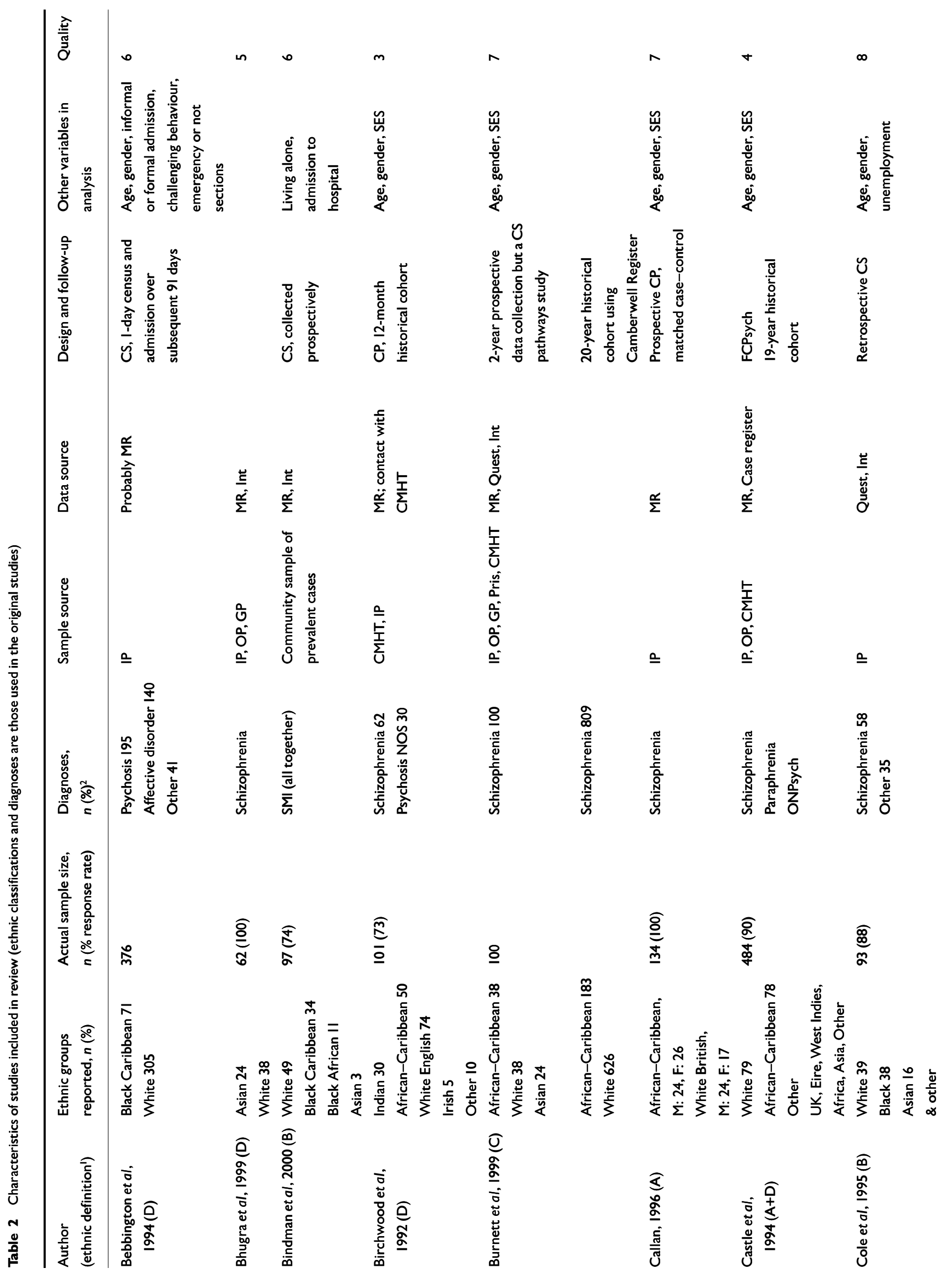




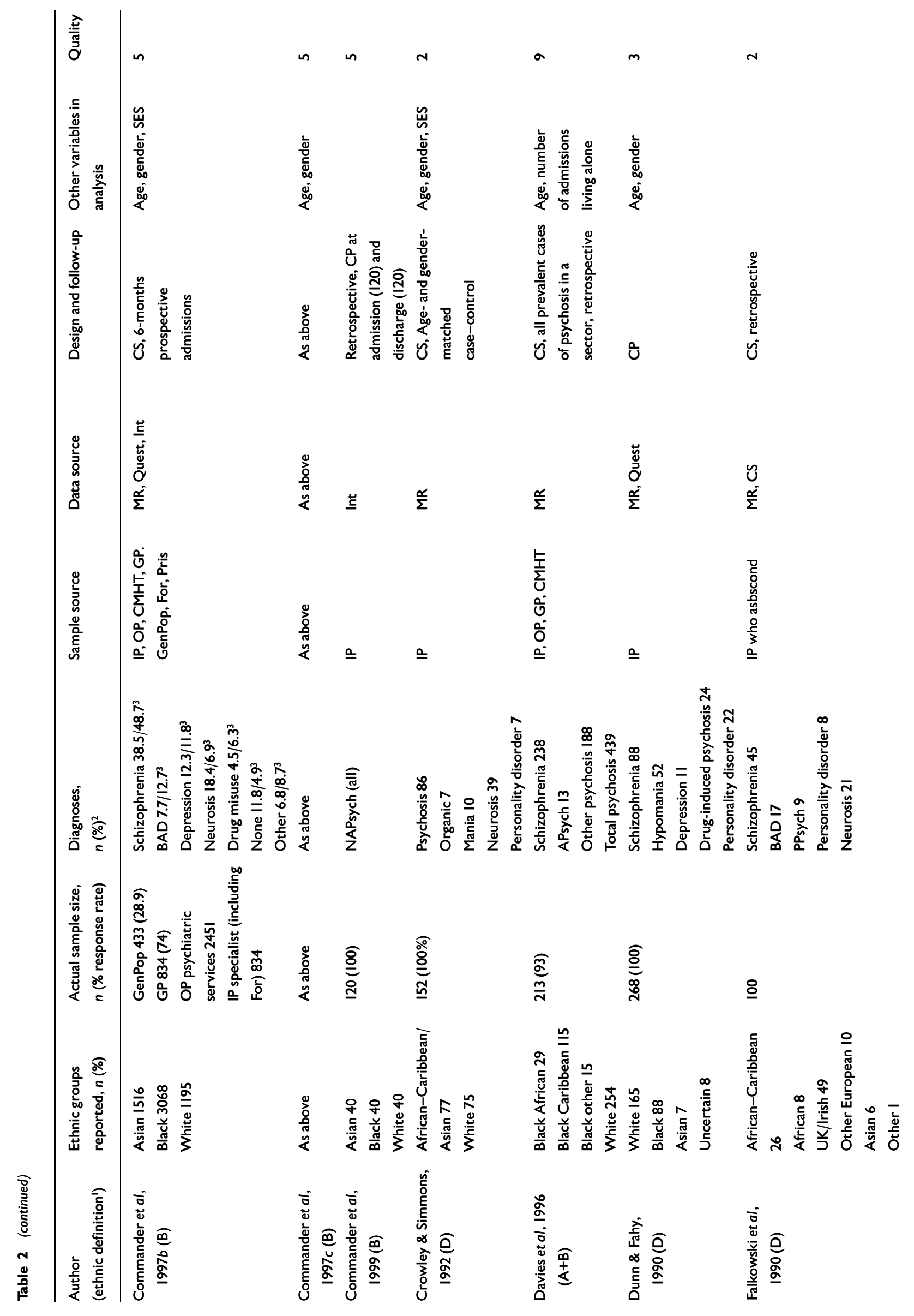




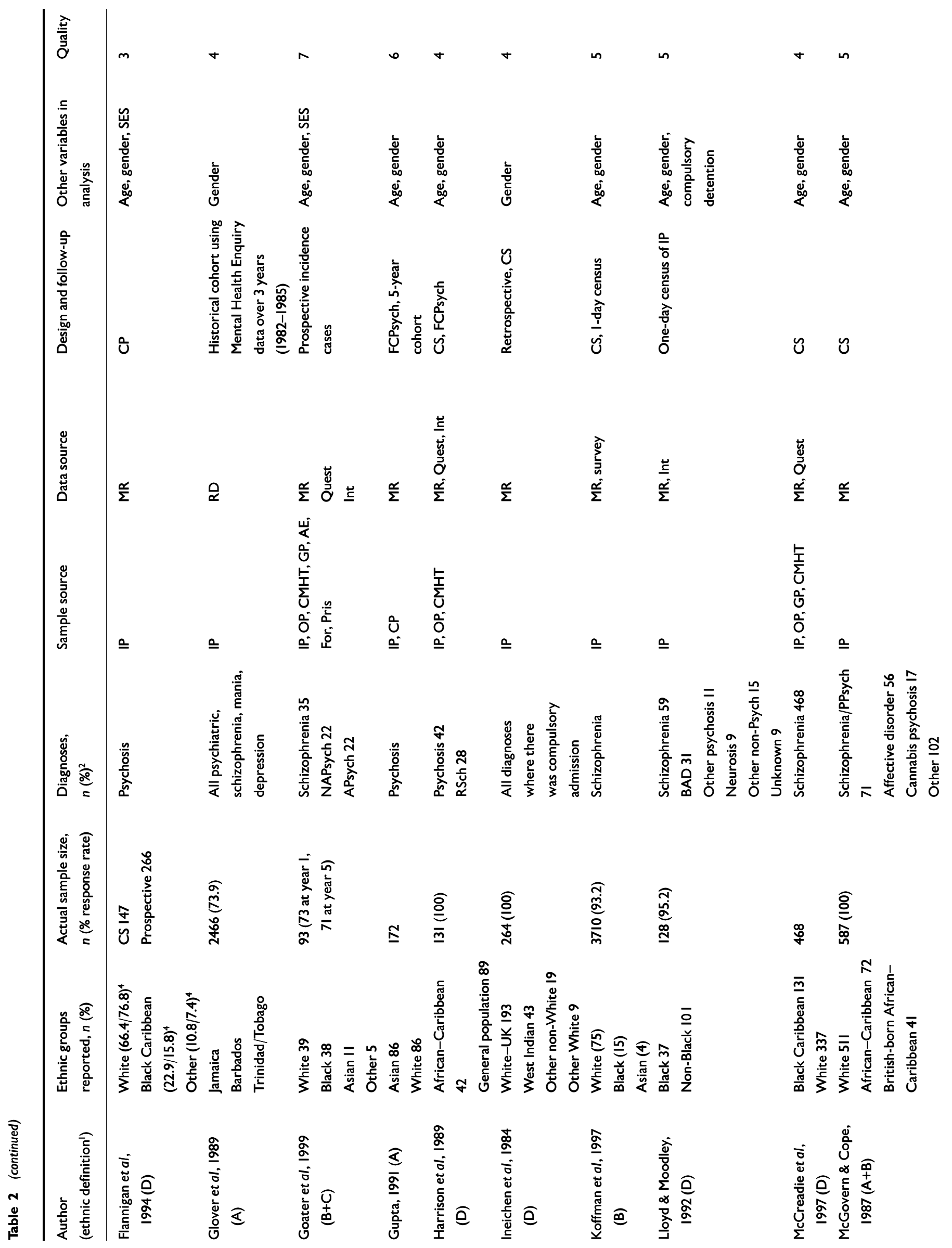




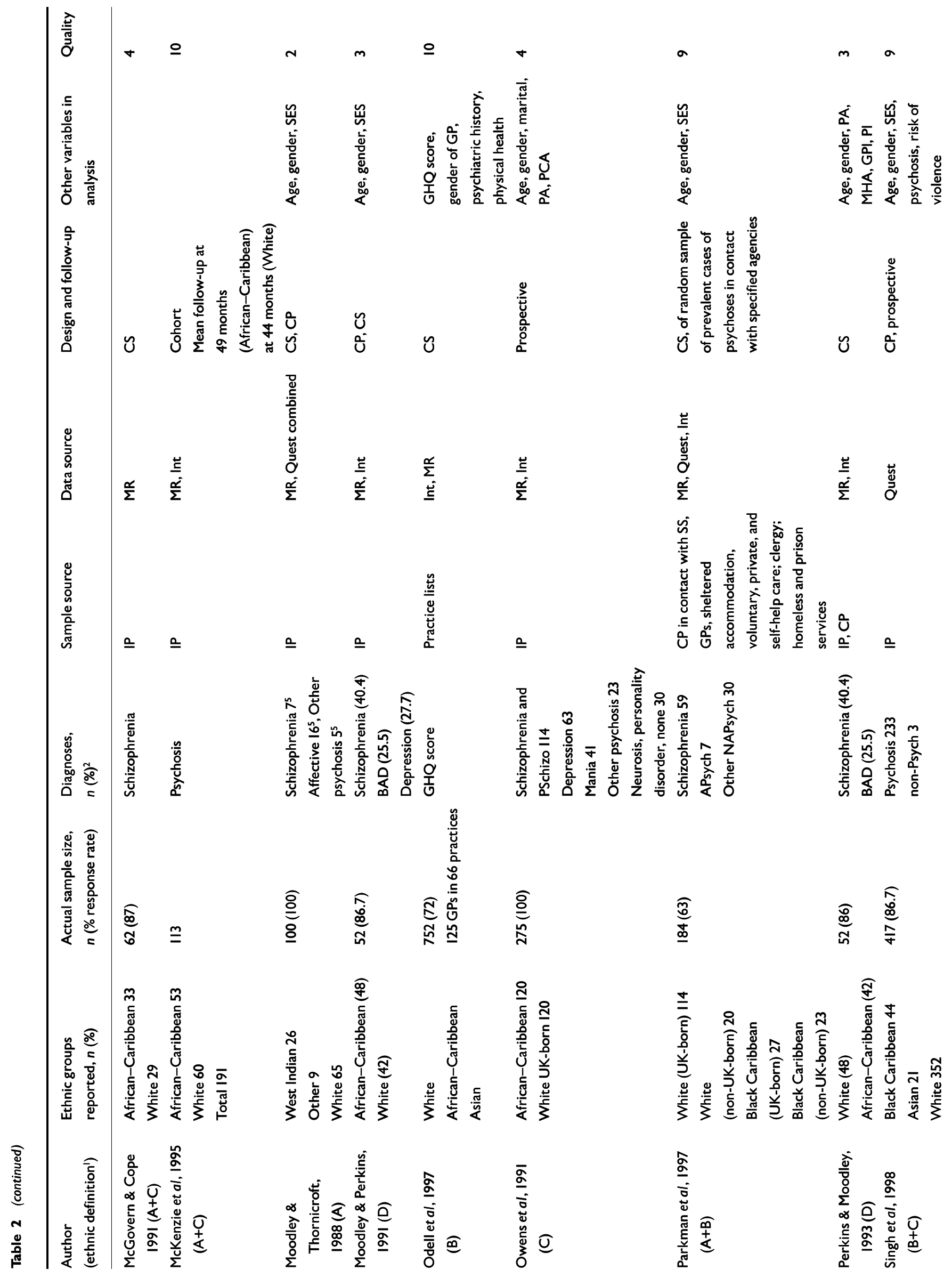




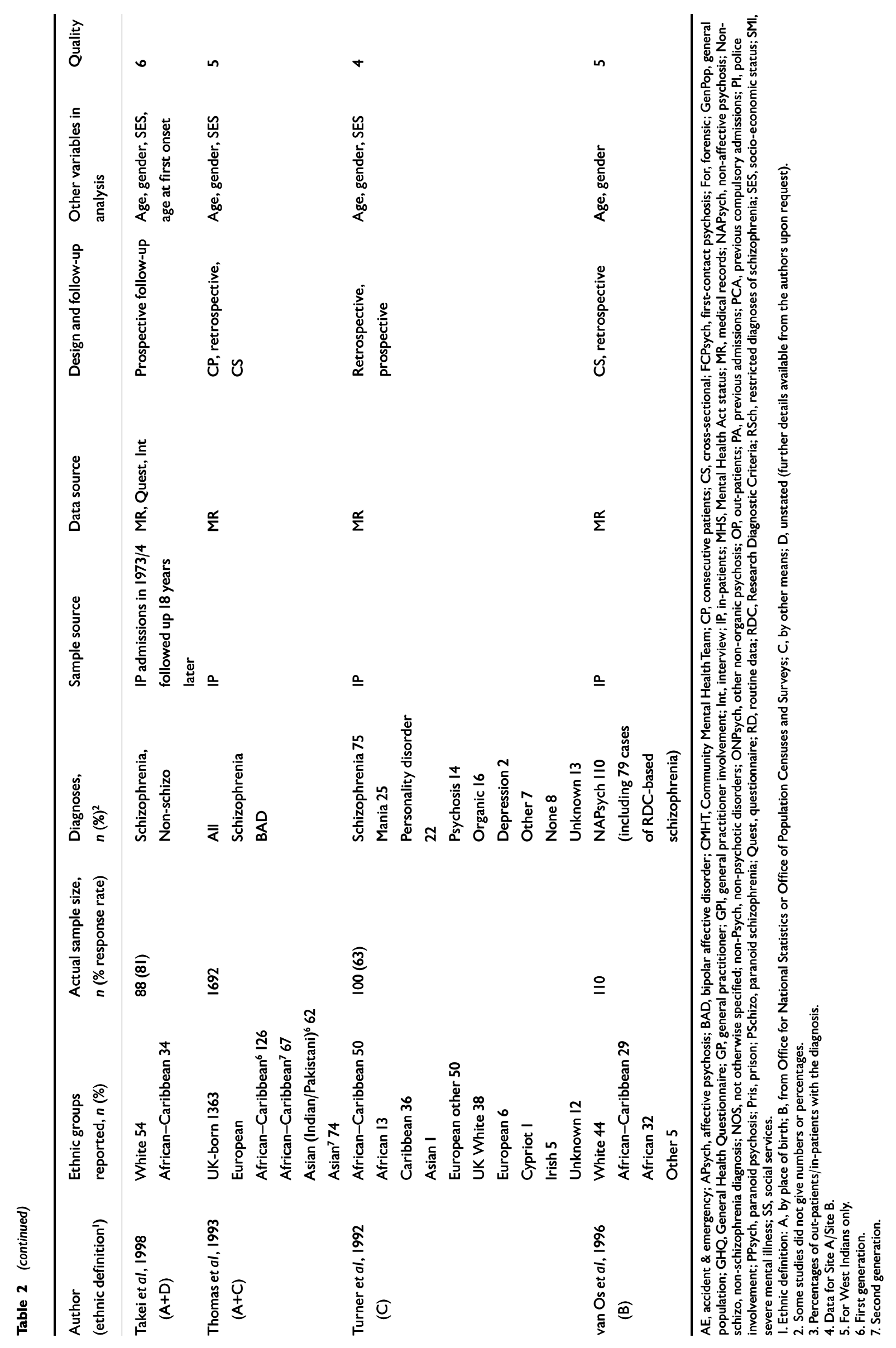


between people of African-Caribbean and African origin. Eight papers included South Asians as a separate category but did not distinguish between people of Pakistani, Indian or Bangladeshi origin. Six papers identified White subgroups but none analysed them separately. Ten papers used country of birth alongside ethnicity information. Nineteen studies did not use ethnic group census categories; studies collecting data before 1991 used proxy measures for ethnic group, such as place of birth. Thirteen papers did not give any explanation for their classification of ethnic groups. Most papers included small numbers of participants from each ethnic group. Twenty-eight papers adjusted for age, 28 for gender and 16 for some measure of socio-economic status, with 24 taking measures to address potential confounding due to variation in diagnoses or illness severity. Although socio-economic status is known to be a confounder in studies of ethnic variations in health, papers adjusting for it did not always score highly on the total quality scores. None of the papers reported sample size calculations. The majority of studies (26) investigated services in London; other locations were Birmingham (McGovern \& Cope, 1987, 1991; Birchwood et al, 1992; Commander et al, 1997a,b, 1999), Manchester (Thomas et al, 1993), Nottingham (Harrison et al, 1989; Owens et al, 1991; Singh et al, 1998) and Bristol (Ineichen et al, 1984).

\section{Do pathways to specialist care vary with ethnic group?}

Black patients had more-complex pathways to specialist services, seeing at least three carers before contact with specialist services (Commander et al, 1999). Compared with White patients, a greater proportion of Black patients had some contact with a helping agency the week before psychiatric service contact (Harrison et al, 1989) and admission was more likely to follow a domiciliary visit (Commander et al, 1997a). Compared with White and South Asian patients who visited their general practitioner (GP), Black people were less likely to be referred to specialist services (Thomas et al, 1993; Cole et al, 1995; Burnett et al, 1999). An explanation might be the GPs' lower likelihood of recognising a psychiatric problem in Black people (Odell et al, 1997; Bhugra et al, 1999; Burnett et al, 1999). However, among patients presenting to general practice who are recognised to have a mental health problem, Black patients were more likely to be found in specialist services (Commander et al, $1997 b, c)$. The police were more likely to be involved in admissions or readmissions of Black people (Thomas et al, 1993; Burnett et al, 1999; Commander et al, 1999). These three papers had medium-quality scores, as did one showing that police involvement before admission was explained by a lack of GP involvement rather than ethnic origin of the patients (Cole et al, 1995). Two medium-quality papers found that Black people were most likely to present in crisis, often seeing the duty psychiatrist in an accident and emergency department as a first point of contact with services (Turner et al, 1992; Cole et al, 1995). In contrast, a low-quality paper showed no variation between ethnic groups in the pathways to specialist care (Moodley \& Perkins, 1991). Variation in the diagnostic categories and differences in ethnic group composition do not explain the heterogeneous outcomes of these studies.

In west London, specialist referral following primary care assessments appeared to be equally common among White and South Asian patients, but hospital admission was more likely among South Asians following a domiciliary visit (Bhugra et al, 1999; Burnett et al, 1999). In Birmingham, South Asians had the highest community rates of mental disorder, were the most frequent consulters in primary care and were less likely than White people to have their mental disorder recognised (Commander et al, 1997b,c). Of all ethnic groups with a mental disorder, South Asians were the least likely to be referred to specialist care (Commander et al, 1997b; Odell et al, 1997). Quality ratings were similar for papers from Birmingham and London and do not explain the different findings. However, the two papers reporting no variation in detection between South Asians and Whites contained smaller samples (total South Asians=24) than studies detecting significant differences (total South Asians=1516).

\section{Is contact between specialist services and Black and South Asian people maintained?}

Compared with White patients, services were less likely to maintain contact with non-Whites in one part of south London (Norwood) but not in another neighbouring area (Nunhead; McCreadie et al,
1997). Another paper from London found no variation between ethnic groups in continuity of community care (Bindman et al, 2000), whereas African-Caribbean people in Birmingham had more 'broken contact' with aftercare services (McGovern \& Cope, 1991). All three of these papers were of medium quality with a cross-sectional design and specifically recruited AfricanCaribbean people. The study with the largest sample size found differences between geographical areas, suggesting that variation in local service configuration and practice are influential and that ethnicity alone does not account for variations in patient contact (McCreadie et al, 1997).

In two papers from London, compared with White people, Black people were more likely to be in contact with services at 5and 18-year follow-up, respectively (Takei et al, 1998; Goater et al, 1999). These papers were of medium quality and both recruited Black people of Caribbean origin. There were no papers that looked specifically at the pattern of South Asian peoples' contact with specialist services.

\section{Use of in-patient services}

Of 20 papers on in-patients, 17 reported measures of in-patient service use (representation on in-patient units (13) consistently showed greater use of in-patient services by Black people (McGovern \& Cope, 1987; Owens et al, 1991; Birchwood et al, 1992; Thomas et al, 1993; Flannigan et al, 1994; Callan, 1996; van Os et al, 1996; Commander et al, 1997b,c; Koffman et al, 1997; McCreadie et al, 1997; Parkman et al, 1997; Takei et al, 1998). One of these positive studies was of high quality and nine were of medium quality. Two papers (of low quality) found no variation in in-patient service use (Ineichen et al, 1984; Castle $e t$ al, 1994). One paper, a first-incidence study, found an excess of in-patient use by White patients (Goater et al, 1999). A study of admissions found an excess of admissions among Black women in one London borough but a $40 \%$ lower admission rate compared with White women in another borough (Bebbington et al, 1994). Quality scores and differences in the ethnic composition of the samples, as far as this could be judged, do not account for this heterogeneity. The evidence most consistently suggests an excess use of in-patient facilities by Black patients. Two papers on first-contact (incident) admissions indicate no excess among Black people (Castle et al, 1994; 
Goater et al, 1999), despite adjustment for age, gender and socio-economic status in one paper (Goater et al, 1999). Among patients of Caribbean origin, Jamaicans had the highest annual admission rates to British hospitals (Glover, 1989).

Despite variation in study quality, geographical region and South Asian group composition and different degrees of adjustment for confounders and diagnosis, results for in-patient use were more consistent for South Asians than for Black patients. Compared with Black patients, South Asians were less likely to be admitted for in-patient care, had the lowest admission rates to secure wards (Commander $e t$ al, 1997c; Koffman et al, 1997), had shorter admissions than other ethnic groups (Gupta, 1991) and were least likely to be readmitted (Birchwood et al, 1992). Compared with White patients, South Asians were more likely to be admitted to inpatient care (Commander et al, 1997b; Koffman et al, 1997).

\section{Compulsory admissions}

Of 23 papers measuring compulsory admissions, the majority (18) showed a higher rate for Black compared with White patients (Ineichen et al, 1984; Moodley \& Thornicroft, 1988; Harrison et al, 1989; Dunn \& Fahy, 1990; Moodley \& Perkins, 1991; Owens et al, 1991; Birchwood et al, 1992; Crowley \& Simmons, 1992; Lloyd \& Moodley, 1992; Perkins \& Moodley, 1993; Thomas et al, 1993; Davies et al, 1996; Koffman et al, 1997; McCreadie et al, 1997; Parkman et al, 1997; Singh et al, 1998; Takei et al, 1998; Commander et al, 1999). Eight of these adjusted for age, gender and socio-economic status. Two adjusted for other potential confounders: class; past admissions; Mental Health Act status (compulsory admission for assessment or detention); police involvement; and general population representation (Perkins \& Moodley, 1993; McKenzie et al, 1995). Other papers adjusted for age at first contact (Takei et al, 1998), psychosis, risk of violence and diagnosis (Singh et al, 1998). One paper that adjusted for firstcontact admissions, past admissions and marital status showed excess admissions among Black people (Owens et al, 1991), challenging reports that the absence of a relative or intimate relationship accounts for compulsory admission (Cole et al, 1995). Davies et al (1996) recruited people with psychosis from a broad range of services.
After adjustment for living alone as well as for age and total number of previous admissions, this paper still found excess compulsory admissions among Black people. Although papers reporting on in-patients suggest that a higher rate of readmissions and absconding explained the excess of compulsory admissions among Black people (Falkowski et al, 1990; Thomas et al, 1993), Davies et al (1996) adjusted for previous admissions and still found higher rates of compulsory admissions among Black people.

Some papers reported contradictory findings. For example, a medium-quality paper showed that, compared with White women, Black women were less likely to be voluntarily admitted in one London borough (South Southwark), but were more likely to be voluntarily admitted in another London borough (Hammersmith \& Fulham; Bebbington et al, 1994). Such differences probably reflect variations in local practice and services. The majority of papers, using a variety of ethnic group measures and study designs and adjusting for a number of potential confounders, provide strong evidence for a relative excess of compulsory admissions of Black people.

Of three papers investigating compulsory admissions among South Asian patients, two low-quality papers found that these were less common among South
Asian patients (Birchwood et al, 1992; Crowley \& Simmons, 1992). One mediumquality paper found no difference between South Asian and White patients (Thomas et al, 1993). These findings are inconclusive as all three studies relied on routinely collected data from medical records and included small numbers of South Asian people.

We calculated a summary odds ratio from the 12 papers (Fig. 1) that reported the exact proportions of compulsorily admitted Black and White in-patients and sample sizes. The meta-analysis gave a pooled odds ratio (Blacks compared with Whites) of 4.31 (95\% CI 3.33-5.58). Analysis of the funnel plot did not show evidence of publication bias $(P=0.69)$.

\section{DISCUSSION}

\section{Main findings}

Of many studies addressing any one of our review questions, few actually used the same measure of outcome; therefore, we did not report estimates because, if presented in isolation, these few estimates are likely to give a biased impression. However, the original data tables are available on request and will be posted on the Queen Mary website. Our review suggests that Black people are overrepresented among

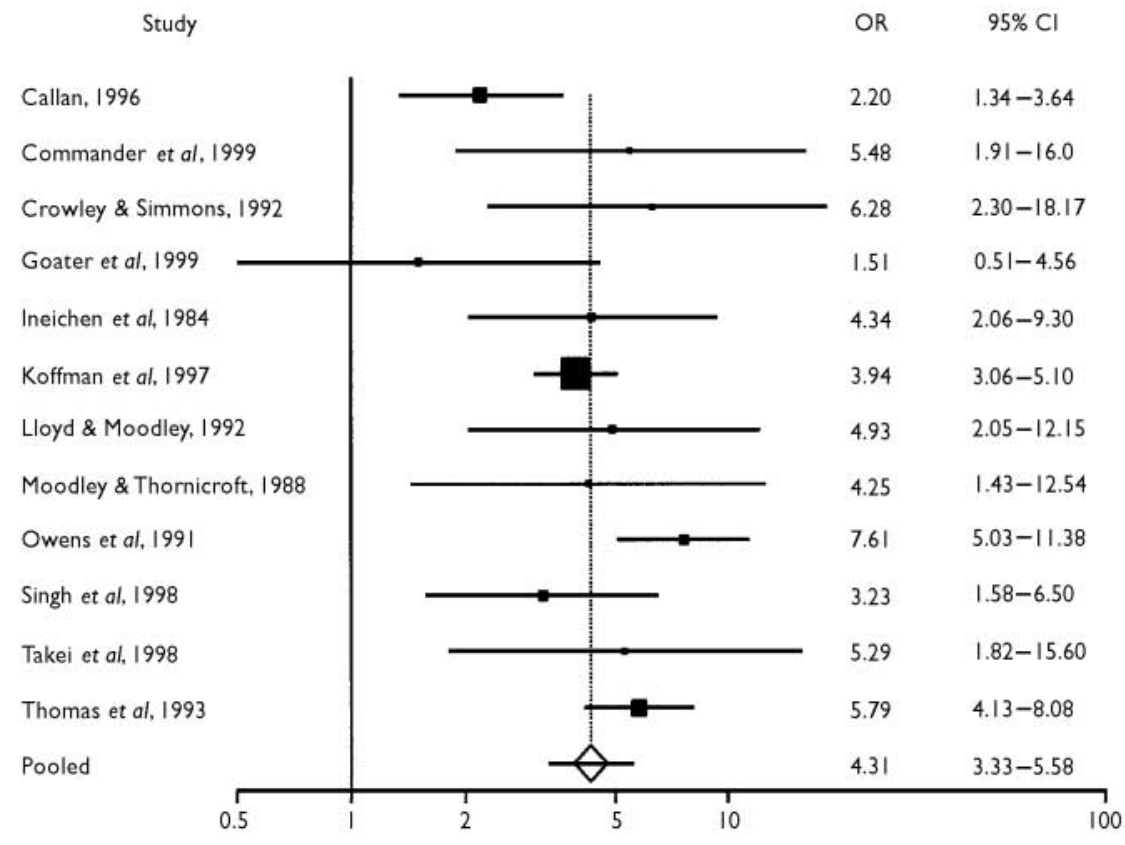

Fig. I Odds ratio meta-analysis plot (random effects), Black $v$. White, from meta-analysis of I2 papers on compulsory admission to in-patient facilities. 
in-patients, and that Asian patients use in-patient facilities less often than do White patients. Unexpectedly, two papers report an excess of voluntary admissions for White people. There is some evidence for variations in pathways to specialist mental health care, with Black people traversing more complex pathways. Variation in primary care assessments or primary care involvement could explain some of the ethnic differences in pathways to specialist services but the primary care literature is small. Despite concerns about Black people falling out of care, two papers showed that they were more likely to be in contact with services after 5 and 18 years. This could reflect effective delivery of necessary care, or professionals' anxieties about perceived risk. Black people on in-patient units were four times more likely to experience a compulsory admission compared with White people. This is consistent with research in forensic and prison services (Bhui et al, 1998; Coid et al, 2000) and with research informing the proposed amendments of the Mental Health Act. These reported twice the risk (Wall et al, 1999) and six times the risk of compulsory detention of Black people (Audini \& Lelliott, 2002).

\section{Explaining the findings}

No papers reported investigations of discrimination as a risk factor. Perceived discrimination could have a detrimental effect on mental health, placing Black people at higher risk of mental illness and perhaps of mental health service use (Karlsen \& Nazroo, 2002). Perceived discrimination within health care services may further compound this, whereas excessive use of compulsory admission could account for less satisfaction with services and fear of contact with services. Although social isolation (Cole et al, 1995) can also mediate higher contact with emergency services among Black patients, local variations in clinical practice and service provision are well recognised as sources of inequalities. Most studies did not compare regional variations of clinical practice or service configuration. Most studies were based in London. Even though the majority of the UK's minority ethnic communities live in the largest cities, mental health policy should reflect the needs of regions with smaller proportions of minority ethnic groups. Indeed, recent evidence suggests that contextual effects, such as a lower ethnic density, can actually lead to higher rates of schizophrenia requiring greater service use (Boydell et al, 2002).

\section{Methodological considerations}

The composition of any one ethnic group varied across the papers. This raises uncertainty about the generalisability of the findings from any single paper to populations that fall within the same ethnic group but have different ethnic subgroup profiles (for example, South Asians with different places of birth, country or region of origin within the Indian subcontinent). Improvement in the quality of the more recent publications is encouraging but the majority did not justify their classification of ethnic groups (Senior \& Bhopal, 1994) and did not take account of possible variations in service use between subgroups within the larger ethnic categories. Adjustment for socio-economic status did not always relate to study quality, but adjustment for confounders generally and ethnic group definitions did relate to quality.

\section{FUTURE PRIORITIES}

Studies including White subgroups and South Asians, services outside of London and community and primary care studies were underrepresented in the literature. Future studies should explore explanations for variations that go beyond assigning all differences to ethnic origins. More-tailored study designs are needed to assess aetiological factors, mechanisms to explain ethnic variations and interventions. Where ethnic differences are evident, future investigations should address potential explanatory factors, such as discrimination, practice variation, service configuration and social isolation. Greater attention to place of birth, religion, duration of residence in the UK, place and level of education and cultural identity could also refine the interpretation of data beyond assigning all differences to ethnic origin.

\section{APPENDIX}

\section{Inclusion and exclusion criteria}

\section{Inclusion criteria}

Variation studies

UK-based studies, measuring variation in service use or provision for mental health care. Report data for more than one ethnic group, retrospective, prospective or cross-sectional studies of use or provision.
Intervention studies

International studies, including quantitative studies of interventions aiming to improve access and uptake of mental health services by different ethnic groups. The types of interventions could include educational (for users and/or health care professionals), organisational, financial, regulatory. Study designs could include controlled trial, parallel group or beforeand-after studies.

\section{Grey/unpublished literature}

UK-based data sought from health authorities and by asking three 'experts' to identify any major omissions from the identified publications.

\section{Service use}

Studies that investigate the rates of use by ethnic groups of facilities provided for mental health care, whether service provision is targeted or nontargeted.

\section{Service provision}

Studies were included that investigate provision of services for different ethnic populations and the uptake of these services by their targeted population.

\section{Management of disorders}

Drug treatment of any affective or psychotic condition in primary or secondary care.

\section{Participants}

Adults identified by ethnic group with an affective or psychotic disorder.

\section{Date of publication}

January 1983 to end October 2000

\section{Language}

English, French or German.

\section{Exclusion criteria}

Studies of services for children, adolescents, psychosexual disorders, substance misuse, alcohol misuse and forensic mental health were excluded.

\section{ACKNOWLEDGEMENTS}

We thank Juliet Formby for carrying out the bibliographic searches, reviewing and coding abstracts and providing administrative support, Alain Besson for designing the search strategies and Marilyn Aviles for contributing to the review protocol and the criteria for judging identification of ethnic groups and reviewing and coding abstracts. For the metaanalysis, we thank Laura Bright for extraction and tabulation data and Helen Tate for performing the analyses.

\section{REFERENCES}

Acheson, D. (1998) Independent Enquiry into Inequalities in Health Report. London: Stationery Office.

Audini, B. \& Lelliott, P. (2002) Age, gender and ethnicity of those detained under Part II of the Mental Health Act 1983. British Journal of Psychiatry, 180, 222-226.

Bebbington, P. E., Feeney, S. T., Flannigan, C. B., et al (1994) Inner London collaborative audit of admissions in two health districts. II: Ethnicity and the use of the 
Mental Health Act. British Journal of Psychiatry, 165 743-759.

Bhugra, D., Corridan, B., Rudge, S., et al (1999) Early manifestations, personality traits and pathways into care for Asian and white first-onset cases of schizophrenia. Social Psychiatry and Psychiatric Epidemiology, 34, 595-599.

Bhui, K., Brown, P., Hardie, T., et al (1998) AfricanCaribbean men remanded to Brixton Prison. Psychiatric and forensic characteristics and outcome of final court appearance. British Journal of Psychiatry, 172, 337-344.

Bindman, J., Johnson, S., Szmukler, G., et al (2000) Continuity of care and clinical outcome: a prospective cohort study. Social Psychiatry and Psychiatric Epidemiology, 35, 242-247.

Birchwood, M., Cochrane, R., Macmillan, F., et a (1992) The influence of ethnicity and family structure on relapse in first-episode schizophrenia. A comparison of Asian, Afro-Caribbean, and White patients. British Journal of Psychiatry, 161, 783-790.

Boydell, J., van Os, J., McKenzie, K., et al (2002) Incidence of schizophrenia in ethnic minorities. I: London: ecological study into interactions with environment. BMJ, 323, 1336-1338.

Burnett, R., Mallett, R., Bhugra, D., et al (1999) The first contact of patients with schizophrenia with psychiatric services: social factors and pathways to care in a multi-ethnic population. Psychological Medicine, 29 475-483.

Callan, A. F. (1996) Schizophrenia in Afro-Caribbean immigrants. Journal of the Royal Society of Medicine, $\mathbf{8 9}$, 253-256.

Castle, D. J., Phelan, M., Wessely, S., et al (1994) Which patients with non-affective functional psychosis are not admitted at first psychiatric contact? British Journal of Psychiatry, 165, 101-106.

Cochrane, R. \& Sashidharan, S. P. (1996) Menta Health and Ethnic Minorities: a Review of the Literature and Implications for Services. NHS Centre for Reviews and Dissemination/Social Policy Research Unit. Report 5. York: University of York.

Coid, J., Kahtan, N., Gault, S., et al (2000) Ethnic differences in admissions to secure forensic psychiatry services. British Journal of Psychiatry, 177, 24I-247.

Cole, E., Leavey, G., King, M., et al (1995) Pathways to care for patients with a first episode of psychosis. A comparison of ethnic groups. British Journal of Psychiatry, 167, 770-776.

Commander, M. J., Odell, S., Sashidharan, S. P., et al (1997a) A comparison of the socio-demographic and clinical characteristics of private household and communal establishment residents in a multi-ethnic inner-city area. Social Psychiatry and Psychiatric Epidemiology, 32, 421-427.

_ , Dharan, S. P. S., Odell, S. M., et al (1997b) Access to mental health care in an inner-city health district. I: Pathways into and within specialist psychiatric services. British Journal of Psychiatry, 170, 312-316.

_ , _ , _ , et al (1997c) Access to mental health care in an inner-city health district. II: Association with demographic factors. British Journal of Psychiatry, $\mathbf{I 7 0}$ 317-320.

\section{_ Cochrane, R., Sashidharan, S. P., et al (1999)}

Mental health care for Asian, black and white patients with non-affective psychoses: pathways to the psychiatric hospital, in-patient and after-care. Social Psychiatry and Psychiatric Epidemiology, 34, 484-49I.

Crowley, J. J. \& Simmons, S. (1992) Mental health, race and ethnicity: a retrospective study of the care of

\section{CLINICAL IMPLICATIONS}

Black patients are more likely to be voluntary or compulsory in-patients.

- Pathways to specialist services are more complex for Black patients, with more crisis routes of entry into care.

- Primary care assessments show ethnic variations that could explain some of the variations in specialist service use.

\section{LIMITATIONS}

- We excluded qualitative studies that might shed light on putative mechanisms that explain our findings.

- The variety of study designs, quality of studies, ethnic group definitions and outcome measures makes comparison, and specifically meta-analyses, inappropriate. This limited the review to a narrative account of differences.

- There is little information on White subgroups, practice and service variations, South Asian groups, services outside of London and primary care and community services.

KAMALDEEP BHUI, MRCPsych, STEPHEN STANSFELD, FRCPsych, Department of Psychiatry, Barts and the London, Queen Mary's School of Medicine and Dentistry, London, UK; SALLY HULL, MD, Department of General Practice and Primary Care, Barts and the London, Queen Mary's School of Medicine and Dentistry, London, UK; STEFAN PRIEBE, MD, FUNKE MOLE, MSc, Department of Psychiatry, Barts and the London, Queen Mary's School of Medicine and Dentistry, London, UK; GENE FEDER, MD, Department of General Practice and Primary Care, Barts and the London, Queen Mary's School of Medicine and Dentistry, London, UK

Correspondence: Kamaldeep Bhui, Department of Psychiatry, Barts and the London, Queen Mary's School of Medicine and Dentistry, Mile End Road, London El 4NS, UK. E-mail: k.s.bhui@mds.qmw.ac.uk

(First received I8 March 2002, revised 25 July 2002, accepted 3I July 2002)

ethnic minorities and whites in a psychiatric unit. Journal of Advanced Nursing, 17, 1078-1087.

\section{Davies, S., Thornicroft, G., Leese M., et al (1996)} Ethnic differences in risk of compulsory psychiatric admission among representative cases of psychosis in London. BMJ, 312, 533-537.

Department of Health (1999) The National Service Framework for Mental Health. London: Department of Health.

— (2000) The NHS Plan. London: Department of Health.

Dunn, J. \& Fahy, T. A. (1990) Police admissions to a psychiatric hospital. Demographic and clinical differences between ethnic groups. British Journal of Psychiatry, I56 373-378.

Falkowski, J., Watts, V., Falkowski, W., et al (1990) Patients leaving hospital without the knowledge or permission of staff - absconding. British Journal of Psychiatry, I56, 488-490.

Flannigan, C. B., Glover, G. R., Feeney, S.T., et al (1994) Inner London collaborative audit of admissions in two health districts. I: Introduction, methods and preliminary findings. British Journal of Psychiatry, $\mathbf{1 6 5}$, 734-742
Glover, G. R. (1989) Differences in psychiatric admission patterns between Caribbeans from different islands. Special Psychiatry and Psychiatric Epidemiology, 24 209-2II.

Goater, N., King, M., Cole, E., et al (1999) Ethnicity and outcome of psychosis. British Journal of Psychiatry, I75, 34-42.

Gupta, S. (1991) Psychosis in migrants from the Indian subcontinent and English-born controls. A preliminary study on the use of psychiatric services. British Journal of Psychiatry, 159, 222-225.

Harrison, G., Holton, A., Neilson, D., et al (1989) Severe mental disorder in Afro-Caribbean patients: some social, demographic and service factors. Psychological Medicine, 19, 683-696.

Ineichen, B., Harrison G. \& Morgan, H. G. (1984) Psychiatric hospital admissions in Bristol. I: Geographical and ethnic factors. British Journal of Psychiatry, 145, 600-604.

Karlsen, S. \& Nazroo, J. (2002) Relation between racial discrimination, social class and health among ethnic minority groups. American Journal of Public Health 92, 624-631

Koffman, J., Fulop, N. J., Pashley, D., et al (1997) Ethnicity and use of acute psychiatric beds: one-day 
survey in North and South Thames regions. British Journal of Psychiatry, I7I, 238-24I.

\section{Lloyd, K. \& Moodley, P. (1992) Psychotropic medication and ethnicity: an inpatient survey. Social Psychiatry and Psychiatric Epidemiology, 27, 95-101.}

McCreadie, R. G., Leese, M., Tilak-Singh, D., et a (1997) Nithsdale, Nunhead, and Norwood: similarities and differences in prevalence of schizophrenia and utilisation of services in rural and urban areas. British Journal of Psychiatry, 170, 31-36.

McGovern, D. \& Cope, R. (1987) First psychiatric admission rates of first and second generation AfroCaribbeans. Social Psychiatry, 22, 139-149.

_ \& _ (1991) Second generation Afro-Caribbeans and young whites with a first admission diagnosis of schizophrenia. Social Psychiatry and Psychiatric Epidemiology, 26, 95-99.

McKenzie, K., van Os, J., Fahy, T., et al (1995)

Psychosis with good prognosis in Afro-Caribbean people now living in the United Kingdom. BMJ, 3II, 1325-1328.

Moodley, P. \& Thornicroft, G. (1988) Ethnic group and compulsory detention. Medicine Science and Law, 28, 324-328.
_ \& Perkins, R. E. (199I) Routes to psychiatric inpatient care in an inner London Borough. Socia Psychiatry and Psychiatric Epidemiology, 26, 47-51.

Odell, S. M., Surtees, P. G., Wainwright, N. W., et al (1997) Determinants of general practitioner recognition of psychological problems in a multi-ethnic inner-city health district. British Journal of Psychiatry, I7I, 537-54I.

Owens, D., Harrison, G. \& Boot, D. (1991) Ethnic factors in voluntary and compulsory admissions. Psychological Medicine, 2I, 185-196.

Parkman, S., Davies, S., Leese, M., et al (1997) Ethnic differences in satisfaction with mental health services among representative people with psychosis in south London: PRiSM study 4. British Journal of Psychiatry, I7I, 260-264.

Perkins, R. E. \& Moodley, P. (1993) Perception of problems in psychiatric inpatients: Denial, race and service usage. Social Psychiatry and Psychiatric Epidemiology, 28, 189-193.

Raine, R. (2000) Does gender bias exist in the use of specialist health care? Journal of Health Services Research and Policy, 5, 237-249.

Senior, P. A. \& Bhopal, R. (1994) Ethnicity as a variable in epidemiological research. BMJ, 309, 327-330.
Singh, S. P., Croudace, T., Beck, A., et al (1998) Perceived ethnicity and the risk of compulsory admission. Social Psychiatry and Psychiatric Epidemiology, 33, 39-44.

Takei, N., Persaud, R., Woodruff, P., et al (1998) First episodes of psychosis in Afro-Caribbean and White people. An 18-year follow-up population-based study. British Journal of Psychiatry, 172, 147-153.

Thomas, C. S., Stone, K., Osborn, M., et al (1993) Psychiatric morbidity and compulsory admission among UK-born Europeans, Afro-Caribbeans and Asians in central Manchester. British Journal of Psychiatry, I63, 91-99.

Turner, T. H., Ness, M. N. \& Imison, C.T. (1992) Mentally disordered persons found in public places. Diagnostic and social aspects of police referrals (Section 136). Psychological Medicine, 22, 765-774.

van Os, J., Castle, D., Takei, N., et al (1996) Psychotic illness in ethnic minorities: clarification from 1991 census. Psychological Medicine, 26, 203-208.

Wall, S., Churchill, R., Hotopf, M., et al (1999) $A$ Systematic Review of Research Relating to the Mental Health Act (1983). London: Department of Health. 